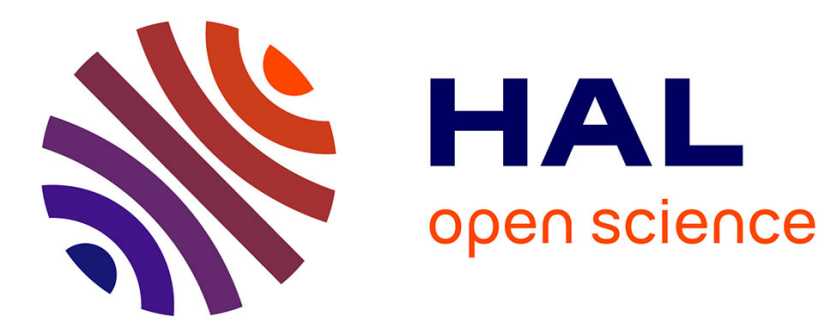

\title{
La question féminine, angle mort de la démocratie islamique en Iran
}

\author{
Fariba Adelkhah
}

\section{To cite this version:}

Fariba Adelkhah. La question féminine, angle mort de la démocratie islamique en Iran. Politix, 2000, 13 (51), pp.143-161. 10.3406/polix.2000.1107 . hal-01011209

\section{HAL Id: hal-01011209 \\ https://hal-sciencespo.archives-ouvertes.fr/hal-01011209}

Submitted on 23 Jun 2014

HAL is a multi-disciplinary open access archive for the deposit and dissemination of scientific research documents, whether they are published or not. The documents may come from teaching and research institutions in France or abroad, or from public or private research centers.
L'archive ouverte pluridisciplinaire HAL, est destinée au dépôt et à la diffusion de documents scientifiques de niveau recherche, publiés ou non, émanant des établissements d'enseignement et de recherche français ou étrangers, des laboratoires publics ou privés. 


\title{
La question féminine, angle mort de la démocratie islamique en
} Iran

In: Politix. Vol. 13, N51. Troisième trimestre 2000. pp. 143-161.

\begin{abstract}
The Women's Cause. An Irrelevant Issue in the Islamic Democracy of Iran Fariba Adelkhah

Since the end of the 80 's, the condition of Iranian women became significantly better. Many of them participate in the public sphere and got an economical or social activity. Rafsandjani's politicy of liberalization furthered this womens's investment, which became more explicitly political. However, women remain out of a number of social areas and are still victims of social and legal discriminations. Indeed, only seem possible the progress respecting the traditionnal Islamic think about women's condition in life, and serving the "honour of the system», rather than attacking it. In this context, the veil paradoxically makes easier the investment of women in the public sphere.
\end{abstract}

\section{Résumé}

La question féminine, angle mort de la démocratie islamique en Iran

Fariba Adelkhah

Dès la fin des années 1980, la situation des femmes iraniennes s'est améliorée. Nombre d'entre elles ont investi l'espace public et ont pu exercer une activité économique ou sociale. La politique de libéralisation menée par Rafsandjani a permis à cet investissement féminin de s'accroître et de prendre une tournure plus explicitement politique. Mais les femmes demeurent exclues de vastes secteurs de la société et continuent à être l'objet de discriminations juridiques et sociales. Les seules avancées possibles ont en effet été celles qui respectaient la pensée islamique traditionnelle de la question féminine, qui servaient l'« honneur du système » plutôt que de le subvertir. C'est ainsi paradoxalement le port du voile qui a favorisé l'inscription des femmes dans la sphère publique.

Citer ce document / Cite this document :

Adelkhah Fariba. La question féminine, angle mort de la démocratie islamique en Iran. In: Politix. Vol. 13, N51. Troisième trimestre 2000. pp. 143-161.

doi : 10.3406/polix.2000.1107

http://www.persee.fr/web/revues/home/prescript/article/polix_0295-2319_2000_num_13_51_1107 


\section{La question féminine, angle mort de la démocratie islamique en Iran}

Fariba ADELKHAH

$\mathrm{E}$ n 1997, deux cent trente-huit Iraniens se portèrent candidats à l'élection présidentielle. Parmi eux figuraient neuf femmes, dont Azam Taleghani, la fille de l'un des inspirateurs les plus prestigieux de la Révolution de 1979, l'ayatollah Mahmoud Taleghani, et fondatrice de la première association de femmes islamiques au lendemain de la Révolution ${ }^{1}$. Cette dernière, dans sa déclaration d'intention, ne cachait pas qu'il s'agissait pour elle de dissiper l'incertitude que recelait l'article 115 de la Constitution, selon lequel la présidence de la République devait revenir à un Rejâl (Homme) du champ religieux et politique : convenait-il de lire un être de sexe masculin ou un être humain, ou encore une personnalité, le terme pouvant être interprété indifféremment de ces trois manières dans le Coran? Le Conseil des gardiens de la Constitution ne retint que quatre candidats, de sexe masculin. Mais, à aucun moment, pour justifier le rejet des neuf candidates et notamment de madame Taleghani, il n'argua de leur féminité, mettant en avant $d^{\prime}$ 'autres raisons ${ }^{2}$. La classe politique et la presse se gardèrent de commenter la mesure, que l'on pouvait lire aussi bien comme une reconnaissance implicite de la possibilité pour une femme d'accéder à la

\footnotetext{
1. La société des femmes de la Révolution islamique d'Iran qui publie depuis 1979 Payâm-e Hadjar, une revue bimensuelle devenue aujourd'hui hebdomadaire après avoir été mensuelle et semestrielle, cf. Zanan, 53, 1378/1999, p. 14-17.

2. Des raisons générales valables pour les deux sexes comme par exemple: le manque d'éducation suffisante, la limite du dévouement à la cause révolutionnaire, le passé antirévolutionnaire, etc.
} 
présidence de la République que comme l'impossibilité pratique de cet événement. Un silence éloquent qui illustre bien l'ambiguité de la condition des femmes en République islamique d'Iran, vingt ans après la Révolution. Elles sont à la fois présentes dans la sphère publique à une échelle sans égal dans la région du Golfe, et bridées ou en tout cas volontiers ignorées dans leur action.

\section{La participation des femmes à l'espace public}

L'élection de Mohammad Khatami à la présidence de la République a semblé ouvrir une nouvelle ère pour les femmes iraniennes, que l'on disait volontiers assujetties et enfermées par l'ordre à la fois archaïque et phallocratique de la République des ayatollahs et par la misogynie de l'islam. Nombre d'entre elles avaient en effet soutenu sa candidature, et sa victoire a paru être la leur en même temps que celle des jeunes. En novembre 1998 l'irruption de quelques centaines de "supporteures" dans le stade Azadi, bien décidées à fêter le retour triomphal d'Australie de l'équipe nationale de football et à enfreindre l'interdiction réglementaire, a été largement saluée comme étant la confirmation de cette évolution.

Mais en réalité cette dernière est bien antérieure à l'ouverture khatamiste et se confond avec l'histoire de la Révolution et de la République. Dès la fin des années 1980, il était clair que le nouveau régime ne pouvait se réduire à une logique binaire entre dominants et dominés, aux premiers rangs desquels auraient figuré les femmes. Paradoxalement le voile avait permis à nombre d'entre elles, issues de la moyenne et petite bourgeoisie, des classes populaires ou des milieux ruraux, d'investir l'espace public et d'exercer une activité économique ou sociale, notamment à la faveur de la guerre. Cet élargissement de leur champ d'action avait été rendu possible par leur mobilisation révolutionnaire et par l'idéologie d'une partie au moins des penseurs islamiques, tels Ali Shariati, l'ayatollah Morteza Motahhari et Mehdi Bazargan. La pointe ou l'avant-garde de la participation sociale des femmes avait pris le visage d'une élite qui se réclamait haut et fort de son engagement islamique et révolutionnaire. De façon plus large et diffuse, c'était bel et bien la condition féminine qui se transformait de façon beaucoup plus profonde que ce qu'avait pu faire la modernisation autoritaire et parfois passablement cosmétique de l'Ancien régime, même si ses acquis ont finalement été assez largement préservés, une fois dépassées les tensions révolutionnaires, grâce à la résistance des femmes, militantes islamiques en tête. La sociabilité des réunions religieuses féminines - les jalesch - était très révélatrice de ces bouleversements. En d'autres termes, l'adhésion des femmes à la République islamique ou tout au moins à certaines de ses politiques et de ses pratiques ne pouvait être sous-estimée, ne serait-ce que parce qu'elle reposait sur des représentations et des 
convictions morales: quoique l'on en contestât le caractère obligatoire, le port du hejâb équivalait à la récusation de la fesâd (la corruption exprimant le dérèglement de la société) et à ce titre légitimait la présence des femmes dans la sphère publique ${ }^{3}$.

Avec la fin de la guerre et la politique de libéralisation conduite par Ali Akbar Hachemi-Rafsandjani, la participation sociale des femmes s'est étendue tout au long des années 1990 et a pris une tournure de plus en plus explicitement politique. Là aussi les dynamiques décisives ont été socioéconomiques: l'éducation des jeunes filles s'est accrue, l'urbanisation s'est accélérée, la transition démographique s'est enclenchée de façon spectaculaire 4 . Déjà, sous la contrainte de la crise économique et de la guerre, les femmes s'étaient adonnées au commerce informel avec les autres pays du Golfe, la Turquie et la Syrie, souvent en profitant d'un pèlerinage ${ }^{5}$. Le trait saillant de ces pratiques est la fusion presque systématique de l'activité marchande ou plutôt contrebandière et de la dévotion ou de la bienfaisance, l'une et l'autre étant susceptibles de servir la distinction et l'affirmation sociale des intéressées.

Dans ce contexte, le passage au politique était à peu près inévitable. Depuis 1979, le Parlement ne comprenait qu'une poignée d'élues (quatre députées durant les trois premières législatures en 1980, en 1984 et en 1988) et, dans le gouvernement, les femmes brillaient par leur absence. Le vrai tournant a sans doute été l'élection législative de 1996. Les candidates ont été plus nombreuses qu'auparavant (82 en 1992, 324 en 1996 et 504 en 2000). Durant la campagne, les femmes ont été très actives et ont fréquemment formé des "Fronts de propagande » (setâdhâh-ye tablighâti), s̀ymétriques à ceux qu'avaient organisés les hommes. Fait notable, cette mobilisation ne s'est pas cantonnée aux grandes villes. Enfin la consultation a été marquée dans une mesure appréciable par la performance inattendue de certaines candidates, telles Jamileh Kadivar, Nahid Shid, Bibi Qodsyeh Alavi, Elaheh Rastgou, Iran Ahou Riya, ou Faezeh Hachemi ${ }^{6}$. Par son brio plus encore que par sa qualité de fille du président de la République, cette dernière s'est imposée sur la scène médiatique et a donné au féminisme islamique un nouveau visage : excellente cavalière, elle est viceprésidente du Conseil national olympique et présidente du Conseil de la solidarité entre les femmes sportives des pays musulmans et elle milite activement en faveur du sport féminin; par ailleurs elle incarne un chic

3. Sur ces questions, of. Adelkhah (A.), La Reiolution sous le zoile. Le's femmes islamique's d'Iran, Paris, Karthala, 1991.

4. Ladier (M.), Population, société et politique. De la monarchie à la République islamique, Thèse de doctorat, Paris, Ecole des hautes études en sciences sociales, 1999.

5. Adelkhah (F.), "Le retour de Sindbad. L'Iran dans le Golfe", Le's ítude's du Ceri, Paris, Ceri/Sciences-l'o, 1999.

6. Adelkhah (F.), Etro moderne' e'n Iran, Paris, Karthala, 1998, p. 138-139.

7. Ce Conseil, entièrement géré par des femmes, est en charge d'organiser des compétitions sportives exclusivement féminines entre les pays musulmans. 
islamique juvénile qui a donné lieu à de véritables effets de mode vestimentaire. Certes, les résultats du scrutin n'ont pas été à la hauteur de la visibilité des candidates et de l'intensité des combats électoraux qu'elles ont menés, puisque seules douze d'entre elles ont été élues. Mais les gros bras de l'organisation populiste et nostalgique de la Révolution, le Ansar-e hezbollah, ne se sont pas trompés sur la signification de l'événement: quelques semaines après le deuxième tour, ils ont attaqué des femmes cyclistes dans le parc Tchitgar que leur avait ouvert le maire réformateur de Téhéran, Gholamhossein Karbastchi, un proche de Faezeh Hachemi.

Premières du genre depuis la Révolution, les élections locales de février 1999 ont confirmé que les femmes étaient désormais vraiment parties prenantes de l'exercice du suffrage universel. Le nombre des candidates s'est élevé à 7000 sur 300000 candidats au total, chiffre évidemment disproportionné mais dont la portée politique a été réelle. Surtout, au-delà de ce déséquilibre, des femmes se sont portées candidates dans des villes saintes, des provinces périphériques ou des circonscriptions rurales - par exemple à Qom, dans le Sistan-Baloutchistan, le Hormozgan et le Khouzistan - dans lesquelles on ne les attendait a priori pas, attestant que la participation sociale ou politique du " deuxième sexe "n'était décidément pas l'apanage des grandes métropoles urbaines ou des classes favorisées. Le fait que seulement 781 femmes ont été élues (sur un total de 200000 conseillers municipaux) ne doit pas oblitérer l'enracinement de ce phénomène dans la société ${ }^{8}$.

D'autant que, répétons-le, celui-ci est porté par les transformations objectives de la condition féminine en matière d'emploi, d'éducation et de maîtrise de la fécondité. Sur ce plan, l'existence d'une presse et d'une édition féminines très vivantes est révélatrice. Des revues comme Farzaneh ${ }^{9}$, Jens-e dovvom (Le deuxième sexe) et des mensuels comme Zanan (Les femmes), Payam-e Zan (Le message de la femme) ou des hebdomadaires comme Zan-e Rouz (Femme actuelle), Payam-Hajar (Le message d'Hadjar) ont animé un véritable débat féminin, en particulier sur les questions juridiques, notamment depuis les dix dernières années, et font désormais partie du paysage médiatique au même titre que les autres périodiques, s'attirant d'ailleurs également les foudres de la justice : Zan, le quotidien créé par Faezeh Hachemi, a lui-même été suspendu quelques mois après sa publication en 1998. Dans le domaine

8. Sur 781 élues, 484 l'ont été dans les milieux ruraux. De plus, dans cinquante-six villes, les candidates se sont trouvées en tête des élus, par exemple à Abadan, Tchalous, Dezfoul, Sari. Dans cinquante-huit villes, elles occupent la seconde position comme par exemple à Ardebil, Ispahan, Khorramshah. Avec la région de Téhéran, celle du Khouzistan a le nombre le plus élevé d'élues, soit vingt-six. Viennent ensuite le Gilan, Ispahan et Kerman. Dans les milieux ruraux, le nombre le plus élevé des élues se trouve d'abord dans la région de Khorassan avec soixante-treize élues, puis celle du Gilan avec cinquante-six, celle de Kerman avec cinquantetrois, celle de Téhéran avec quarante-huit, et celle du Sistan-Baloutchistan avec quarante-deux. Cf. Zanan, 52, 1378/1999, p. 62.

9. Prénom persan de femme, qui signifie femme de grande vertu. 
de l'édition les femmes sont également très présentes - plus de trente éditrices tiennent des stands à la foire annuelle du livre à Téhéran privilégiant pour certaines la publication des cuvres féminines. Il existe également des femmes cinéastes de réputation internationale ${ }^{10}$ ou des romancières auteurs de best-sellers. Enfin les étudiantes représentent plus de $40 \%$ de l'effectif universitaire et sont dans certaines branches, comme la médecine, majoritaires ${ }^{11}$.

Sous la pression de cette participation sociale des femmes, des débats juridiques et politiques qu'elle a inspirés, et d'une vie culturelle spécifique, y compris au sein du champ religieux, le discours des hommes a dû changer. Déjà l'ayatollah Khomeyni, qui s'interrogeait sur la légitimité du droit de vote accordé aux femmes par le Shah en 1963 et qui plus probablement le contestait au fond de lui-même ${ }^{12}$, ne l'a pas remis en cause au lendemain de la Révolution de 1979 et ne manquait pas de recevoir des délégations de citoyennes en corps constitués. Aujourd'hui nul ne doute plus de la nécessité de la participation des femmes à la vie sociale et politique, pas même chez les conservateurs, même si les modalités de cette dernière sont sujettes à débat et cristallisent la ligne de partage entre les grands courants factionnels. Quelle que soit leur obédience, les hommes politiques et les responsables administratifs ont des femmes pour collaboratrices ou conseillères, traitent les questions relevant de la condition féminine comme n'importe quel

10. Habibian (M.), «Under Wraps on the Stage: Women in ther Performing Arts in PostRevolutionary Iran", Cahiers du Cemoti, 28, 1999.

11. «Vaziyat-e zanân az 1355 tâ 1375 [La situation des femmes entre 1976 et 1996]", Hamshahrir, 16 mai 1376/1997; «Bist sâl âmouzesh-é 'âli [Vingt ans d'enseignement supérieur]», Jâme'eh va Dâneshlgâh, 22 décembre 1377/1998.

12. Trois livres réunissent, en reprenant chacun à sa façon, les points de vue de l'imam Khomeyni entre $1341 / 1962$ et $1361 / 1982$ sur les femmes. Toutefois, il est difficile de trouver une réponse directe à la question qui nous préoccupe, car elle n'est jamais abordée en ellemême mais est mise en relation avec d'autres questions brûlantes de l'époque, aux yeux du Guide de la Révolution. Résumons-les. Il y a d'abord le constat que toutes les propositions concernant la réforme de la Constitution au nom du progrès et de la liberté ne sont que des prétextes pour camoufler les véritables intentions du pouvoir despotique en vue d'amoindrir l'islam. Ensuite la question du droit de vote pour les femmes est considérée comme non prioritaire par rapport à des problèmes cruciaux qu'affronte la société, tels que la pauvreté et la misère. Enfin, l'entrée des femmes dans un tel Parlement n'aura pour conséquence que la corruption du fait même de la mixité qu'elle exige. Par exemple on trouve les passages suivants: "C'est pour nous empêcher de voir les vrais problèmes que pose l'application de la Constitution depuis le début du siècle, que l'Etat nous jette l'os de la participation féminine aux élections. La participation de la moitié de la population à la vie de la société, c'est bien là de belles phraséologies séduisantes qui n'ont d'autres effets que la corruption et la prostitution "; «Le récent décret de l'Etat quant à la participation des femmes dans les élections est sans fondement du point de vue religieux et abrogé du point de vue constitutionnel ", Dar jost-o jouy-e râh dar kalâm-e emâm [A la recherche de la direction au travers des paroles de l'imam], Téhéran, Amir Kabir, 1361/1382; Zan az didgâlté c'mâm khomeyni lLa femme du point de vue de l'imam Khomeynit], Téhéran, Nehzat-zanân-e mosalmân, 1360/1980; Simây-e zan dar kalâm-e emâm Khomeyni [La figure de la femme dans le discours de l'imam Khomeyni], Téhéran, Vezârat-e ershâd, $1364 / 1985$. 
dossier de politique publique, ne manquent pas de tenir le discours obligé sur la place des femmes dans la République islamique, célèbrent chaque année l'inévitable journée de la femme qui coïncide avec l'anniversaire de la fille du Prophète, et priment des « femmes exemplaires » tout en saluant leur dévouement à la cause de la République. Les femmes, quant à elles, exercent en théorie leur devoir civique sur un pied d'égalité avec les hommes : il est révélateur que personne parmi les clercs n'ait songé à soutenir que leurs voix, ou, au sein du Parlement, celles des députés de sexe féminin devraient valoir la moitié de celles des hommes, en application du précepte coranique qui veut que le témoignage d'une femme ne vaut que la moitié de celui de $1^{\prime}$ homme ${ }^{13}$. Simultanément, pourtant, cette banalisation de la participation sociale et politique des femmes donne lieu à de rudes conflits qui opposent les différents courants. Non pas que les conservateurs soient nécessairement en la matière des obscurantistes : par exemple, dans l'un de leurs fiefs, la Chambre de commerce et de l'industrie de Téhéran, les employées ont eu depuis longtemps une liberté de mise, certes relative, mais sans commune mesure avec ce qu'autorisaient bien des administrations ou des entreprises publiques contrôlées par la gauche. Simplement, au niveau des représentations, l'idée d'une droite rétrograde s'est imposée au moins dans certains milieux intellectuels, et les prises de position de quelques-uns de ses leaders, de pair avec la pudibonderie des clercs traditionalistes qui en sont souvent proches, y ont aidé. Ainsi, lors du débat parlementaire sur la proposition de loi concernant la réévaluation de la dot en fonction de la crise économique, Abbas Abbasi, le député de Bandar Abbas, n'a pas hésité à gracieusement faire valoir qu'un éventuel remboursement de celle-ci par le mari en cas de répudiation de l'épouse devait tenir compte de la dépréciation de la femme du fait de son âge ! Il est cependant instructif que ce type de propos ne soit pas payant: le député en question a durement été pris à parti sur ce point pendant la campagne électorale de février 2000 et n'a pas été réélu ${ }^{14}$.

Cette guerre de représentations entre la droite et la gauche tend à se focaliser sur la pratique du hejâb. Lors de la campagne présidentielle de 1997 la rumeur voulait ainsi qu'Ali Akbar Nategh Nouri, le champion des conservateurs, imposerait en cas de victoire le port obligatoire du tchador, plus strict et plus contraignant encore que le simple foulard ou même que le maghnaeh en usage dans les administrations et les établissements scolaires. Tant et si bien que l'intéressé dut démentir solennellement ce mauvais bruit. De façon quotidienne, le zèle plus ou moins grand que les forces de l'ordre

13. A propos de l'ambiguïté et de l'incohérence du statut juridique des femmes, cf. Ebadi (S.), Zymaran (M.), Sonnat ia tajaddod dar hoquq-e iran [La tradition et la modernité des lois en Iran], Téhéran, Ganjeh Dânesh, 1375/1996.

14. Cependant, les résultats des élections de la ville de Bandar Abbas ayant été annulées récemment par le Conseil des gardiens de la constitution, A. Abbasi pourra une nouvelle fois tenter sa chance. 
déploient pour veiller au respect des normes vestimentaires islamiques dans les grandes villes est un bon baromètre du climat politique dans le pays, les cycles de répression alternant avec les cycles de relâchement.

En définitive la République islamique s'est largement construite autour d'un équilibre: elle reconnaît et même promeut la participation sociale et politique des femmes qui sont littéralement omniprésentes dans son discours, voire dans ses non-dits, mais, dans le même temps, elle assigne à celles-ci des limites strictes que révèle bien l'intangibilité de l'obligation du voile, jamais remise officiellement en cause et dont le caractère sacré apparaît comme la dernière digue du régime, tant sur le plan intérieur que dans le domaine de sa politique étrangère. Même les relations avec les EtatsUnis, voire avec Israël, n'inspirent pas une telle fidélité au credo de départ de la République islamique.

\section{La question des femmes et les aléas du processus de régulation politique}

Et c'est bien là que le bât blesse. Si la réduction de la République islamique à un régime d'exclusion sociale des femmes est de toute évidence un contresens, trop fréquemment commis, et si cette dernière repose bien, entre autres, sur la mobilisation d'une dynamique sociale féminine sans égale dans le Golfe persique, il ne faut pas pour autant s'aveugler sur les limites, les ambiguités et les incertitudes d'un tel processus. De ce point de vue, les attentes parfois naïves qu'a suscitées l'arrivée au pouvoir de Mohammad Khatami ne doivent pas tromper. Venant après la percée des élections de 1996, les résultats des municipales de 1999 et des législatives de février 2000 ont révélé la faiblesse du mouvement féminin, y compris dans sa composante islamique stricto sensu sur laquelle nous avions attiré l'attention en 1991. Dans les conseils municipaux élus l'année dernière, les femmes, rappelons-le, ne sont que 781 sur un total de 200000 conseillers, soit moins de $1 \%$. Pareillement, le nouveau Parlement ne compte que 12 élues contre 14 dans l'assemblée sortante et, fait peut-être plus préoccupant encore, celles-ci appartiennent toutes au même milieu social de la petite bourgeoisie intellectuelle des enseignants et des médecins. Six d'entre elles représentent la circonscription de Téhéran, les autres ayant été élues dans les principales métropoles régionales - exception, qui devrait confirmer la règle, faite du cas de Mme Hamideh Edalat, élue à Dashtestan dans la province de Boushehr. Ce qui est apparu comme un élément de progrès aux yeux de certains observateurs - l'appartenance de ces députées à la mouvance réformatrice - trahit plutôt à nos yeux les limites de la représentativité sociale des femmes engagées en politique, qui restent largement les porte-parole de fractions bien circonscrites des classes moyennes urbaines. De vastes secteurs de la société ne connaissent toujours pas d'expression politique féminine, quand bien même les femmes s'y montrent très actives, comme par exemple dans le domaine du commerce informel, dans certaines activités de service, 
dans l'économie de la bienfaisance ou dans le champ religieux. Par ailleurs il n'y a toujours pas de femme ministre au sein du gouvernement plus de vingt ans après la Révolution, même si la présidence de la République comporte depuis Hachemi Rafsandjani des conseillères, pas plus qu'il n'y a de préfet, de maire, de diplomate de sexe féminin bien qu'aucune loi ne l'exclue. Enfin, socialement et même souvent juridiquement, les femmes continuent de faire l'objet de toute une série de discriminations que la commission des droits de l'homme des Nations unies relève chaque année : leur majorité pénale est fixée à 9 ans (contre 14 pour les garçons); elles sont soumises à la tutelle administrative de leur mari; le métier de juge leur reste interdit malgré l'ampleur des débats autour de la nécessité de leur présence dans les tribunaux civils; les stages ou les missions à l'étranger sont rendus problématiques alternativement par le statut de célibataire ou par la condition d'épouse des impétrantes; la publication de photographies de femmes dans la presse est soumise à d'invraisemblables restrictions sous le prétexte du refus de leur marchandisation; et, last but not least, la voix des femmes est interdite de chant dans l'espace public, la seule des restrictions apportées par l'Imam Khomeyni à l'usage de la musique qui soit encore en vigueur ${ }^{15}$.

A cet égard, Mohammad Khatami n'a pas franchement fait mieux que son prédécesseur et n'a pas eu, au sujet de la condition féminine, les gestes ou les discours fondateurs qui ont caractérisé son action réformatrice dans d'autres domaines. Dans sa bouche, les femmes restent à jamais idéalisées à l'image de la fille du prophète, Fatemeh/Zahra ; elles sont censées être des mères, garantes de la santé de la société que doivent continuer de contrôler les hommes. La presse de gauche est curieusement très timorée sur ce thème. Si elle a pris vigoureusement parti dans les débats juridiques en faveur de l'amélioration du statut des femmes ou plutôt des mères, elle s'est abstenue jusqu'à présent de le poser en termes explicitement politiques : alors qu'elle n'a pas craint d'ouvrir le débat sur les modalités du principe constitutionnel du velayat-e faqih (gouvernement du jurisconsulte), elle est muette en ce qui concerne l'obligation du hejâb qui n'est prévue ni par la Constitution ni même par la loi. Paradoxalement le député conservateur Mohammad Javad Laridjani, membre de la commission des affaires étrangères, s'est montré beaucoup plus audacieux pendant la dernière campagne législative.

"Notre jeunesse est-elle dépourvue d'ambition ou déprimée?", lui demandait gravement un journaliste lors de la dernière campagne législative, en février 2000. Notre homme est un intellectuel, qui appartient à une grande famille très influente aussi bien dans le clergé que dans la classe politique. Mais sa réponse n'eut rien de convenu. Souriant, voire enjoué, si l'on en croit les photos de l'hebdomadaire Iran-e javan, il expliqua que cette jeunesse iranienne est bel et bien "bloquée ", et ce essentiellement parce qu'elle n'est pas en mesure de réaliser ses besoins sexuels, et donc de se réaliser. Son

15. Cahiers du Cemoti, 11, 1991. 
argumentation mérite d'être largement reprise tant elle peut surprendre dans la bouche d'un élu conservateur, surtout connu pour ses prises de position sur la politique étrangère. Il rend d'abord hommage à Freud dont l'approche "scientifique" démontre que la répression des besoins sexuels, de la concupiscence a des conséquences inévitables sur la santé physique et mentale des individus : "Si vous n'accédez pas à la richesse à laquelle vous aspirez, vous déprimerez moins que si vos besoins sexuels ne sont pas assouvis. " Laridjani rappelle également l'importance, pour Freud, de la neutralisation des tabous: "Cela veut dire que si nous souhaitons des relations sexuelles rationnelles entre filles et garçons, nous devons les "détabouiser". " Autrement dit, il faut d'abord admettre que ces besoins et ces relations sont naturels, à condition de définir leurs limites normales et rationnelles: "A mon avis, bien des comportements condamnables, la mauvaise humeur chez les adultes ou les excès sexuels chez certaines personnes, procèdent de la répression des besoins sexuels. Bien des attitudes violentes chez les gens ou dans leurs discours sont liées à ce noeud dissimulé dans leur vie". Et de citer Max Weber, pour qui "l'essence de la modernité réside dans le fait que les gens pensent qu'ils font partie d'eux mêmes, qu'ils sont eux-mêmes avant de penser qu'ils appartiennent à une société "; "Nous considérons que la concupiscence est un pêché alors qu'elle est un don de Dieu. Nous la réduisons à sa fonction de reproduction. Même pour la reproduction, si les relations sexuelles étaient exemptes de désir, elles seraient fades et personne ne les rechercherait. Sans satisfaction sexuelle il n'y a pas de paix dans la vie ». Laridjani pourfend alors un autre préjugé : celui qui a trait à la prétendue supériorité de l'homme sur la femme. De ce point de vue, la notion de chasteté est fondamentale. En islam elle ne se limite ni aux relations sexuelles proprement dites, ni aux femmes, alors que dans la société on ne parle de chasteté qu'à propos de la femme qui attend son mari à la maison et n'a pas d'autres "fantaisies" (sic !): "Cette acception n'a rien d'islamique. Elle s'explique par le fait que l'homme veut garder la mainmise sur la femme, veut que la femme soit son bien. La chasteté est un outil de domination masculine. C'est ce que dit Michel Foucault à propos de l'éthique bourgeoise dans L'Histoire de la sexualité. " Ne nous méprenons pas. Laridjani n'est pas en train de s'exprimer dans l'équivalent iranien du Magazine littéraire, ni même dans celui du Nouvel Observateur, mais dans un hebdomadaire de large public, destiné aux jeunes et plutôt marqué à droite ${ }^{16}$. En outre il s'agit d'un discours très politique de candidat, et non de ratiocinations intellectuelles. A preuve son refus de traiter de façon idéaliste de cette question de la sexualité des jeunes. A ses yeux la République islamique ne pourra pas résoudre le problème, comme le préconisent certains, en développant les prêts d'aide au mariage afin de faciliter celui-ci, car elle n'en a tout simplement pas les moyens financiers. Il en appelle à l'autonomie des individus et à la "détabouisation". Il récuse l'imposition de modèles préfabriqués de bonheur et de réussite. Par exemple il recommande que l'on mette fin à la séparation des sexes dans les établissements universitaires,

16. Cf. Iran-e jazân [Le jeune Iran], fév rier 1999. 
puisque l'expérience prouve que le taux de réussite est plus élevé dans les universités mixtes. Ce beau programme n'a pas permis à Laridjani d'être élu.

Mais cette façon très ouverte de parler de la sexualité et de l'égalité des sexes montre qu'en la matière la gauche dite réformatrice n'a pas le monopole de l'innovation. Déjà Hachemi Rafsandjani s'était distingué dès les années 1980 par des prêches évoquant ces questions - y compris, en termes voilés, la nécessaire satisfaction des besoins sexuels des épouses des martyrs de guerre - puis en 1990, en prenant la défense du mariage temporaire, seul à même de satisfaire en toute légitimité islamique les attentes sexuelles de la jeunesse

Ce qui frappe au fond, vingt ans après la Révolution, c'est l'absence de renouvellement de la pensée islamique eu égard à la question féminine. Sur ce plan les années 1970 avaient été dominées, nous l'avons vu, par les ceuvres de l'intellectuel engagé Ali Shariati - auteur du livre culte Fatemel est Fatemeh - et surtout du théologien Morteza Motahhari, dont deux des ouvrages, Le système des droits de la fenme e'n islam et La question du hejâb, jouissaient d'une grande réputation et étaient volontiers commentés dans les jaleseh. Ces deux penseurs ont disparu prématurément et nul ne les a véritablement remplacés pour approfondir cette problématique de l'islam au féminin, à moins de paraphraser leurs thèses. Or ces neuvres sont datées. Shariati s'en tient à une position militante qui n'émeut plus personne. Motahhari, quant à lui, se livre à l'explication des normes mais ne s'interroge pas sur leur fondement historique ni sur l'émergence des nouvelles exigences éthiques, sociales ou politiques qui ne pouvaient pas même frôler son esprit. Il est par exemple impossible de trouver dans ces écrits des réponses aux incertitudes de l'époque: une femme peut-elle devenir président de la République? Comment fixer l'âge du devoir ${ }^{17}$, quand le fiqh l'établit à neuf ans pour les filles mais aussi quand la vie moderne introduit d'autres critères tels que des considérations de santé liées aux conditions climatiques ou la nécessité de préparer des examens scolaires? Comment justifier la tutelle légale ou administrative des hommes sur les enfants et les femmes quand ces dernières sont chefs de famille, célibataires, divorcées ou tout simplement provisoirement séparées de leur mari parti travailler à l'étranger ou à l'autre bout du pays, et quand la famille elle-même est devenue nucléaire? L'ceuvre de Motahhari n'aborde pas ces problèmes, ne serait-ce que parce qu'ils étaient, pour certains, impensables il y a quelques décennies. Ces lacunes sont d'autant plus criantes que les femmes ellesmêmes, largement scolarisées, ont investi l'université et jusqu'aux écoles théologiques, privant le clergé de son monopole en la matière. Ainsi les débats que ces dernières lancent, par exemple à propos de l'âge du devoir, du mariage temporaire, de la polygamie, et qu'elles formulent en termes

17. L'âge d'accomplissement des devoirs religieux, fixé pour les garçons à quatorze ans et pour les filles à neuf ans, et qui équivaut à la majorité pénale. Sur ces questions, cf. Ebadi (S.), Zeymaran (M.), Somnat va tajaddod ..., op. cit. 
scientifiques et rationalistes, ne rencontrent pas vraiment d'écho auprès de la hiérarchie religieuse, dont le silence sur ces sujets est d'autant plus assourdissant qu'elle ne cesse de prendre position sur de multiples faits de société ou points de droit et qu'elle publie plus que jamais.

Politiquement il n'y a pas véritablement d'autonomisation du féminisme islamique, ne serait-ce que parce que les candidatures féminines ne s'émancipent pas d'un jeu factionnel qui est le monopole des hommes. Dans ce contexte, les réformateurs ne manquent pas de demander aux femmes de veiller scrupuleusement à leur hejâb ou de faire preuve de modération dans leurs revendications afin de ne pas donner des armes à leurs adversaires. La droite, elle, surenchérit dans le port très strict du hejâb afin de se démarquer des tendances qui, à ses yeux, trahissent les principes islamicorévolutionnaires de la République. Quand on voit comment les parties prenantes du régime sont parvenues à évincer en février 2000 la mouvance des indépendants qui s'était électoralement imposée en 1996, puis lors des municipales en 1999, on peut douter de la possibilité d'une affirmation politique spécifique des femmes au cour des institutions. La détermination des tenants du pouvoir à sauvegarder "l'honneur du système " - selon la formule consacrée - i.e. son unité, laisse peu de place à une représentation sexuée du politique.

Les femmes engagées en politique sont elles-mêmes très ambiguës ou frileuses à ce propos. Le principe du hejâb semble désormais intériorisé et suscite une adhésion à peu près unanime de leur part. Les déclarations des candidates les plus en vue lors des législatives de février 2000 en sont une bonne illustration. A la question de savoir si le hejâb peut faire l'objet d'un référendum, Jamileh Kadivar, la seconde sur la liste des élus de Téhéran, nous répondra: «Le fondement du hejâb, absolument pas, mais quant à sa forme, il $n^{\prime} y$ a jamais eu d'obligation la concernant. Vous pouvez aujourd'hui trouver toutes les formes du hejâb. Les députés qui vont être élus ont été sélectionnés en fonction de leur foi religieuse. Ils sont croyants et ne vont pas remettre en cause un tel principe qui fait partie des fondements de $l^{\prime}$ islam ${ }^{18}$. " Par ailleurs tout un courant du féminisme islamique - incarné notamment par Madame Taleghani et son journal Payam-c Hadjar - récuse explicitement une approche en termes de gender qui éloignerait les femmes des véritables problèmes de la société tout en les marginalisant.

A la limite, la participation sociale et politique des femmes procède elle aussi de ce fameux "honneur du système ", plutôt qu'elle ne le subvertit. Elle ne se départit pas de sa langue de bois et de ses rituels. La libéralisation politique qui a entraîné une efflorescence du débat social dans la plupart des domaines ne l'a pas vraiment ouvert pour ce qui concerne les questions féminines. Ici il faut bien admettre que le principe du hejâab, dont on peut

18. Source : enquête réalisée par l'auteur à Téhéran en février 2000. 
évidemment penser qu'il est éminemment compatible avec la mobilisation d'un mouvement féminin authentique et qu'il fait l'objet d'une large acceptation du fait de la relation de "confiance"- le terme est souvent utilisé $^{19}$ - qu'il a instaurée entre les gens, a été enfermé dans une problématique restrictive et "légitime " de la condition féminine et n'a pas permis la formulation de revendications autrement plus audacieuses.

Sous le voile, les femmes se sont cantonnées à l'ordre des «tactiques » pour reprendre une expression de Michel de Certeau et ne sont pas parvenues à développer une véritable "stratégie», à se constituer en "lieu propre ». D'une certaine manière, Faezeh Hachemi, en insistant sur la pratique du sport ou sur de nouvelles formes de hejâb, mieux adaptées à la vie moderne et active ainsi qu'au goût du jour, a confusément senti l'importance du mouvement, de la "circulation » pour la transformation de la condition féminine. Mais dans la fureur de la campagne électorale de février 2000, la portée de sa démarche a été ignorée, comme le montre le commentaire de cette étudiante sur le port du tailleur à la place du manteau islamique : «Ce sont des questions marginales pour moi. Il faudrait d'abord que l'on nous traite comme un être humain ayant des droits. On verra ensuite pour le choix du manteau ou du tailleur. C'est d'ailleurs une question de goût $\mathrm{t}^{20} \gg$. En outre le principe du hejâb est toujours susceptible de fonctionner comme une machine de délégitimation du comportement féminin aux mains des hommes, qui ne sont pas naturellement enclins à abandonner leurs privilèges et leur pouvoir. Evidence, dira-t-on, puisque justement le hejâb est censé être un facteur d'exclusion sociale. Mais la complication provient de ce que, historiquement, il ne l'a pas été à un moment précis de la vie du pays et qu'il a favorisé l'inscription des femmes dans la sphère publique, souvent contre la volonté du père, du mari, des frères, voire de certaines autorités religieuses $^{21}$. Insistons sur ce point. C'est bien après avoir contribué à créer une situation irréversible de présence massive des femmes dans le social que l'arme mobilisatrice du voile a été désamorcée, à la fois du fait des faiblesses

19. F. Hachemi déclare : «De façon rationnelle je n'accepte pas que le hejâb soit une obligation. Mais je crois que dans le contexte de notre société le hejâb a été plutôt positif pour les femmes. La raison en est simple. $80 \%$ des gens dans notre société sont des traditionalistes ou des religieuses, si on écarte le nord de la ville de Téhéran. L'obligation du voile a créé une sorte de confiance entre les femmes et la société. Elle a permis que les femmes et les filles participent aux activités sociales. C'est ce qui a permis que la participation sociale des femmes devienne générale, y compris dans les coins éloignés. Le hejấb a permis l'évolution générale des femmes dans la société et il a été utile de ce point de vue. Mais je ne vois pas d'obstacle à un référendum sur la question puisque d'un point de vue rationnel l'obligation du voile n'est pas fondée. " (Entretien avec l'auteur.)

20. Entretien avec l'auteur.

21. La présence des femmes dans la sphère publique, agitant leur poing hors leur tchador, scandant des slogans et marchant côte à côte avec les hommes, était considérée par une bonne partie des dignitaires religieux comme le comble même de la corruption conduisant la société directement à la porte de l'Enfer. 
intrinsèques du mouvement féminin et de l'exacerbation de la crise politique, de la guerre et du durcissement du régime qui s'en est suivi.

Cette fausse contradiction s'éclairera si l'on prend en considération une situation précise, celle de la circonscription électorale de Fariman, dans la région du Khorassan, que nous avions déjà observée lors des législatives de 1996. En février 2000, nous n'avons pas retrouvé cette impression de voir les femmes s'affirmer au cour du processus électoral, ce qui nous avait frappé quatre ans auparavant alors que nous travaillions sur les "fronts de propagande ». Certes, lors de la dernière consultation, aucune candidate ne s'était présentée dans cette ville, assez traditionaliste bien que la gauche y soit solidement implantée. Dans ces conditions, la candidature de Madame Ameneh Beyraghdar, sceur de martyr, mariée et responsable de l'encadrement religieux dans un lycée, pouvait paraître confirmer la tendance générale d'une participation politique croissante des femmes. Cependant les circonstances de son initiative amènent à tempérer un tel jugement. Hossein Ghazizadeh, le leader historique de la gauche dans la région, avait demandé à plusieurs de ses partisans de faire eux-mêmes acte de candidature afin de se substituer à lui au cas où le Conseil des Gardiens de la Constitution l'écarterait de la compétition. Ameneh Beyraghdar faisait précisément partie de ce petit noyau de pressentis. De fait Hossein Ghazizadeh ne fut pas retenu. Il demanda alors à Jafar Afghahi de le représenter et il appela à voter pour lui, vraisemblablement en partie parce que le milieu social dont il est issu, sa trajectoire personnelle, ses idéaux et ses valeurs - qu'ils soient ou non révolutionnaires - l'empêchèrent de faire équipe avec une femme. Néanmoins Mme Beyraghdar choisit de rester en lice, estimant qu'elle méritait de reprendre le flambeau de la gauche dans la ville et espérant bénéficier d'une partie de ses réseaux. En dépit de son courage, de son dynamisme et du soutien de sa famille, notamment de son mari qui est allé jusqu'à vendre sa maison afin de faire face aux dépenses exigées par sa candidature, elle se trouva vite isolée. Les notables locaux négligèrent de relayer sa campagne et apportèrent leur soutien à Jafar Afghahi ou plutôt au candidat virtuel qu'il incarnait, Hossein Ghazizadeh. Faute d'argent et de bonnes volontés prêtes à se mettre à son service en nombre suffisant, elle ne parvint pas à faire entendre sa voix. Et ce d'autant moins que, pour éviter les dépenses excessives, la loi électorale limitait le nombre des stands de propagande à un par candidat dans les petites villes: il n'eût pas été convenable que les femmes se mêlent aux hommes et de facto elles ont déserté les réunions publiques de propagande, cantonnant leur action aux jaleseh (les réunions religieuses) et aux visites familiales. De même les commerçants n'apposèrent sur leur devanture que les affiches des trois ou quatre principaux candidats selon leur propre orientation, et ne laissèrent aucune place à Madame Beyraghdar, pas plus qu'à l'autre candidate, Madame Tahereh Gorji, infirmière, célibataire et également soeur de martyr, 
qui n'avait aucune représentativité particulière et dont les méchantes langues disaient qu'elle était surtout à la recherche d'un époux.

Enfin la circonscription de Fariman comprend la ville frontalière de Sarakhs, à majorité sunnite, marquée par une histoire de vives luttes des ouvriers agricoles à l'encontre de l'une des institutions les plus puissantes de la République, l'Astan-e Qods (institution de biens de mainmorte), et aujourd'hui transformée en zone franche vouée au transit des marchandises entre Dubaï et l'Asie centrale. Il était à peu près impensable pour une candidate de faire campagne à quatre heures de route de son domicile et de dormir dans des lieux collectifs tels que les mosquées 22 . Il apparaît donc que les femmes ne sont pas des candidates à part entière et ne concourent pas à armes égales, au moins en dehors des grandes villes. A la limite, elles font campagne par procuration, par l'intermédiaire de leurs proches de sexe masculin. Le score de Ameneh Beyraghdar et de Tahereh Gorji, à Fariman, en est un triste témoignage: elles ont respectivement obtenu 1600 et 1300 voix sur 110000 suffrages exprimés. La première d'entre elles a juste réussi à mettre en ballottage Jafar Afghahi, son principal rival. Quant à Madame Gorji, elle ne semble même pas avoir recueilli les suffrages de sa propre famille, selon la rumeur ! Au fond, les femmes constituent dans de tels contextes une sorte de piétaille électorale, utile pour activer les réseaux familiaux ou ethniques qui demeurent l'une des clefs des consultations. Vu le score souvent très bas obtenu par la majorité des femmes, on peut aussi se demander si la candidature aux élections locales ou législatives de certaines d'entre elles n'avait pas pour objectif réel de se faire socialement reconnaître pour obtenir une amélioration de leur situation professionnelle dans l'administration - singulièrement dans l'Education nationale - ou pour se constituer un capital symbolique à toutes fins utiles. Autrement dit, la participation politique des femmes est susceptible de contribuer à leur distinction sociale, mais non véritablement à leur accès au pouvoir, même si on peut déjà parler de leur accession à une certaine influence dont les tenants et les aboutissants ne sont pas encore clairement définis. Néanmoins - les aléas de la candidature de Madame Beyraghdar le démontrent - les obstacles à leur percée sur la scène électorale relèvent moins de l'islam ou de la législation républicaine que de contraintes économiques, de normes coutumières et du jeu des alliances, qu'elles soient ou non traditionnelles, entre les notables locaux, dans des contextes qui demeurent profondément marqués par de fortes inégalités dans le contrôle des richesses (terre, puits, positions commerciales oligopolistiques).

22. Comme anthropologue, nous avons nous même rencontré des obstacles de ce genre pour suivre la campagne: les candidats ne pensaient pas pouvoir nous laisser une place dans leur voiture et avançaient immédiatement l'objection du logement ou celle de la compagnie des hommes. 
Bien sûr les choses sont différentes dans les grandes métropoles. Mais y compris là, force est de reconnaître que le féminisme islamique manque de dirigeantes d'envergure en dehors, pour une brève période, de Faezeh Hachemi, qui a été battue aux dernières élections, soit qu'elle a payé son audace, soit qu'elle a été la victime collatérale de la violente campagne dont son père a été la cible. Quelle que soit l'aura de cette dernière, le fait est en lui-même révélateur. Faezeh Hachemi se serait-elle imposée si elle n'avait pas été la fille du président de la République ? Ne peut-on en dire autant de Jamileh Kadivar, l'épouse d'Ataollah Mohajerani, le ministre très en vue de la Guidance et de la culture islamique, et la soeur du théologien dissident Mohsen Kadivar? Non que l'on puisse réduire leur audience aux soutiens familiaux dont elles ont bénéficié. Mais il est clair que leur parcours a été facilité par ces derniers et que les conditions que doivent affronter les candidates de base ne sont pas comparables.

En bref, la participation sociale des femmes, dont la République islamique a été le véhicule paradoxal, reste largement en deçà du politique. Elle reflète avant tout des mutations structurelles de la société iranienne telles que l'urbanisation, la scolarisation, l'élargissement du marché du travail aux femmes du fait de la crise et de la ségrégation professionnelle des sexes dans certains secteurs comme la santé et l'éducation nationale. Elle n'est que marginalement tributaire de l'idéologie politique stricto sensu, malgré le rôle que les femmes ont eu pendant la Révolution de 1979 et l'hommage qui leur est rendu de façon passablement stéréotypée. Elle se traduit surtout par la montée en puissance d'un mouvement évergétique spécifique, par des activités commerciales informelles et par la fin du monopole masculin de l'expression religieuse, vieux de quatorze siècles.

Il n'est pas sûr que deux des principales dynamiques contemporaines, la poussée démographique et l'émigration, modifient les données du problème dans les années à venir. D'une part, les rangs serrés des adolescentes qui rentrent dans l'âge des jeunes adultes paraissent dans leur ensemble assez conformistes, à l'image de la société dans laquelle elles vivent: elle se montrent plus concernées par la quête du prince charmant et par les joies de la consommation que par le féminisme, que celui-ci soit islamique ou non. D'autre part, la diaspora installée dans le Golfe, en Turquie, en Europe occidentale et surtout aux Etats-Unis, est elle aussi foncièrement conservatrice, voire néotraditionnaliste. Sa grande affaire semble être la reconstitution d'une identité iranienne forte dont la famille reste le pivot, tant et si bien que l'on peut parfois se demander s'il ne lui faudrait pas inventer la République islamique si celle-ci n'existait pas ! Il est peu probable que les changements culturels ou, dans une moindre mesure, économiques qu'engendrent les échanges entre l'Iran et l'émigration s'accompagnent d'effets comparables dans le domaine de la condition féminine, même si des membres de la diaspora, en visite en Iran, peuvent afficher un certain 
libéralisme en matière de mœurs comme marque nécessaire de leur distinction sociale vis-à-vis de leurs parents demeurés au pays.

L'ambivalence de la condition féminine en Iran révèle celle des principes islamiques de la Révolution de 1979, et de l'islam lui-même. En l'occurrence, une telle polysémie renvoie à la survivance de deux traditions. Celle de la société iranienne antérieure à l'avènement de la République, dont l'un des répertoires d'action privilégiés, celui du javânntard (homme de bien), est précisément pétri d'ambivalence ${ }^{23}$ et est l'un des fondements des relations sociales tant dans le privé que dans le public. Celle également de l'institution cléricale, qui a toujours résisté à l'homogénéisation du dogme, comme l'ont prouvé l'absence de toute doctrine religieuse de référence, codifiée par un corpus de textes officiels, depuis la Révolution et la floraison d'une abondante littérature théologique publiée par différents établissements d'enseignement, revues ou maisons d'édition.

Si échec de l'islam politique ${ }^{24}$ il y a, c'est, dans le cas de l'Iran, dans cette incapacité ou cette non-volonté d'imposer une telle ligne. L'un des pères de la Révolution, l'ayatollah Mahmoud Taleghani, avait ainsi intitulé son exégèse de la deuxième sourate, Baghara, "une lueur de Coran" pour signifier qu'il avait de l'islam une approche herméneutique ouverte à d'autres interprétations. Qu'elle fût due à sa lucidité philosophique ou à son mysticisme ou encore à sa modestie de javânmard, c'était sans aucun doute une vision non politique, voire peu compatible avec l'exercice du pouvoir. Sa mort l'en a de toute façon dispensé. Mais les dirigeants de la République - et l'imâm Khomeyni lui-même - dans leur souci de centralisation, ne sont pas parvenus, si tant est qu'ils en aient eu l'intention, à supprimer le pluralisme religieux au sein de l'institution cléricale chiite.

Pour autant, on ne peut parler d'un échec du système politique islamique en Iran. D'abord parce que la République est maintenant âgée de vingt-deux ans, ce qui représente une période de stabilité institutionnelle assez considérable dans l'histoire moderne du pays malgré des circonstances néfastes (guerre contre l'Irak, terrorisme des Modjahedin du peuple, crise économique et sanctions internationales) : l'imam Khomeyni est mort en Iran et non en exil, à l'encontre des deux souverains Pahlavi, et la classe politique aux commandes est celle qui a fait la Révolution. Ensuite parce que l'idée d'échec suppose que préexistait un projet originel. Or tel n'a pas été le cas historiquement, de l'aveu des leaders révolutionnaires eux-mêmes. Tous les grands débats idéologiques ou politiques, par exemple relatifs à la notion d'économie islamique ou au velâyat-e faqih, sont postérieurs à la Révolution. En cela le processus d'islamisation est assez comparable à celui de la démocratisation. Il ne porte pas sur la mise en place d'un modèle donné. Il

23. Adelkhah (F.), Etre moderne en Iran, op. cit., chapitre 2.

24. Roy (O.), L'échec de l'islam politique, Paris, Le Seuil, 1992. 
s'apparente à l'exploration d'une voie, à la formation d'un système dont on identifie l'aspiration fondatrice, mais dont les modalités restent à découvrir et à définir. De ce point de vue, il ne faut pas se méprendre sur la signification de la revendication islamique véhiculée par la Révolution. Cette dernière ne visait pas à instaurer un nouveau mode de production, sauf peut-être pour une partie de la gauche islamique nourrie de marxisme, dont les espérances ont vite été déçues avec l'étiolement de la réforme agraire à partir de 1983. Sa visée était plutôt d'ordre durkheimien, si l'on peut dire. Il s'agissait d'assurer la régulation des normes en vue de sauvegarder l'équilibre de la société. Tel était (et reste) le sens de la dénonciation de la corruption (fesâd) et de l'exigence de justice (hagh). L'une et l'autre ne désignaient pas une catégorie précise de la population, du type des "cidevant " ou des " ennemis de classe ", mais respectivement un dérèglement de la société ou la condition de sa bonne santé, même si, dans les faits, la détention de la richesse, toutes classes confondues, faisait (et continue de faire dans une moindre mesure) l'objet d'une certaine suspicion ${ }^{25}$.

25. Il est vrai que, sans qu'il y ait forcément échec de l'islam politique, on peut se poser la question d'un défaut d'hégémonie de cet islam politique dans un pays comme l'Iran. Pour la raison que nous venons d'indiquer la République $n^{\prime}$ a pas rompu avec l'économie politique rentière qu'elle a héritée de l'Ancien régime. Certes, elle promeut un nouveau "type d'homme " (ou de femme) comme nous avons essayé de le montrer dans notre livre (Etre moderne en Iran, op. cit.). Les figures des enseignant(e)s, des universitaires, des journalistes et des étudiant(e)s qui sont des protagonistes centraux des luttes politiques, en sont des incarnations parfaites. Mais ce "style de vie", outre le fait qu'il ne rompt pas forcément avec l'éthique de $l^{\prime}$ " être en société " de l'Ancien régime, ne repose sur aucune base productive nouvelle et n'est pas autonome par rapport aux forces sociales traditionnelles telles que le bazar. En d'autres termes l'échec hégémonique de l'islam politique serait d'abord économique. Il s'exprimerait dans l'incapacité du régime à mettre en ceuvre un ajustement structurel conséquent. Par ailleurs on retrouve là l'ambivalence du hejâb. On a vu que ce dernier est l'un des derniers symboles islamiques de la République. D'où la violence des polémiques idéologiques et des affrontements politiques que suscitent les différentes modalités de sa pratique effective. Mais dans le même temps le hejấb est un symbole dont la portée n'est pas universelle au sein de la nation. Une bonne part de la diaspora n'y adhère pas. Les minorités chrétienne, juive, zoroastrienne, bahai ne s'y reconnaissent pas non plus, sans parler des laïcistes. Il n'est pas non plus sûr que les $15 \%$ de la population iranienne qui sont de confession sunnite le voient d'un ceil favorable dans la mesure où les pays de la région qui peuvent leur servir de référence - la Turquie, l'Irak, le Pakistan et jusqu' aux Emirats arabes unis - ne l'imposent pas. Enfin, parmi la majorité chiite, les femmes islamiques qui le portent volontiers commencent à en récuser éthiquement l'obligation. En définitive, le port du hejâb correspond au style de vie d'une couche bien précise de la population iranienne : celui du "type d'homme" (ou de femme) dont l'ascension a fourni la base sociale du régime. Aussi n'est-il pas étonnant qu'il n'ait jamais fait l'objet d'une disposition constitutionnelle ou légale. Comme symbole de légitimation il s'est vu assigner une mission impossible au regard des réalités du pays. Ce qui pose un problème plus fondamental : une hégémonie islamique ou plus exactement islamo-chiite, est-elle envisageable dans une société aussi hétérogène que l'Iran, et dès lors que la principale autorité doctrinale, à savoir l'institution cléricale, ne peut (ou parfois ne se soucie pas) d'apporter des réponses aux interrogations du monde moderne? Telle est au fond la principale aporie qui entrave la formation d'une "démocratie islamique " et qu'exacerbe la question féminine, plus encore que l'ajustement difficile entre la légitimité démocratique et la légitimité religieuse prévu par la Constitution de 1979. A cet égard l'échec de l'islam politique dont parle $O$. Roy concerne 
Dès lors, dans les faits, l'Etat islamique consiste largement en la survivance des normes traditionnelles de régulation sociale, en la capacité de reproduction ou d'accommodement des élites locales et des grandes forces sociales, et en la très grande flexibilité des principes islamiques au regard des pratiques sociales concrètes, avec ce que cela comporte de disparité dans le temps et dans l'espace. Sur ce plan on ne peut qu'être d'accord avec Olivier Carré26 lorsqu'il souligne l'inexistence de toute contradiction théologique et philosophique entre l'islam et la laicité. Notre seul point de divergence avec cet auteur aurait trait à son interprétation de la "prison scripturaire " dans laquelle l'islam enfermerait les femmes. En réalité, nous l'avons vu, les Iraniennes, avec ou sans le Coran, ne se sont pas laissé emprisonner et les difficultés qu'elles rencontrent dans leur action tiennent moins (ou autant) à l'idéologie ou à la législation de la République islamique, qu'au poids des structures et des représentations culturelles de la société qui se nourrissent du conformisme ambiant.

De ce point de vue le processus démocratique reflète à son tour l'ambivalence de la condition féminine en Iran. Cette dernière, fût-ce sous le couvert du symbole supposé de son assujettissement, le voile, reste l'aune à laquelle peut être évaluée la nature politique de la République islamique. Non qu'il faille exagérer l'importance du problème : après tout la démocratie française ou suisse a pu exclure les femmes du jeu électoral jusqu'à une période somme toute récente, sans que cela mette en cause la nature démocratique du système politique. Mais aujourd'hui l'Iran n'a plus d'islamique, dans les apparences, que le hejâb. Dans ces conditions, l'instauration d'une véritable démocratie islamique, à laquelle disent s'employer les protagonistes de la vie politique, dépend moins des luttes factionnelles et des jeux d'une élite soucieuse de sauver $l^{\prime}$ «honneur du système " dont elle gère les intérêts que de l'extension du débat démocratique aux femmes. Ces dernières ont investi au prix de lourds sacrifices des pans entiers de la société iranienne dont l'importance est peutêtre aussi grande que celle de la scène politique, maintenant que l'Etat se doit d'être "minimum " et n'est plus en mesure de tenir son rang de redistributeur et de rémunérateur. L'un des défis auxquels est confrontée la République islamique est l'adaptation de ses institutions et des modalités de la représentation politique aux transformations très rapides de la société. Le mouvement féminin constitue l'une de ces dynamiques, qu'il faudra bien un jour reconnaître politiquement. Madame Beyraghdar avait placé au-dessus de son stand de propagande, sur la place publique de Fariman, le slogan suivant: «Nous ne voulons pas de tutelle». Elle s'en prenait ainsi à l'adoubement de Jafar Afghahi par l'un des hommes forts de la région,

décidément moins les institutions ou l'Etat eux mêmes que la difficulté à imposer une acception unique de la religion (quand on est musulman).

26. Carré (O.), Islam laïue ou le retour à la grande tradition, Paris, Colin, 1993. 
Hossein Ghazizadeh. Mais dans sa bouche l'allusion à d'autres tutelles, celle des notables sur la vie politique, celle des hommes sur les femmes, était limpide. Madame Beyraghdar a été électoralement battue, certes, mais elle a mis en question les normes sociales et les relations de pouvoir qui l'ont vaincue, tout en mettant fin au monopole des hommes sur la scène politique de cette petite ville conservatrice de province. 